\title{
Ultra Slim and Small UHF RFID Tag Design for Mounting on Curved Surfaces
}

\author{
Carlos David Morales Peña ${ }^{\mathrm{a}}$, Diogo Batista de Oliveira ${ }^{\mathrm{a}}$, Elson José da \\ Silva $^{a}$, Maurício Weber Benjó da Silva ${ }^{b}$ \\ ${ }^{a}$ Graduate Program in Electrical Engineering, Federal Univesity of Minas Gerais, Belo \\ Horizonte, Brazil. \\ ${ }^{b}$ Telecommunications Engineering Department, Fluminense Federal University, Niterói, \\ Brazil.
}

\begin{abstract}
This paper proposes a UHF flexible passive tag antenna for Radio Frequency Identification (RFID) which operates at 902-928 MHz frequency band. As main contribution, we design a electrically small and slim tag antenna by using a flexible commercial substrate. The small antenna has dimensions of $13 \mathrm{~mm}$ x $28 \mathrm{~mm} \times 0.35 \mathrm{~mm}$. The geometry of the tag antenna was optimized, using a Genetic Algorithm, to maximize its read range. The antenna performance was evaluated on flat and curve surfaces, by means of simulations of input impedance, reflection coefficient, gain and read range. Measurements of reflection coefficient were also developed. Results prove that the tag antenna can be wrapped on cylindrical surfaces with read ranges above one meter, suitable for applications related to industrial asset management, access control and identification of small objects such as hardware tools, tin cans, spray bottles and tin PET cylindrical containers.
\end{abstract}

Keywords: Elecrically small antenna, Flexible substrate, Optimization, RFID.

Email addresses: davidmoralesp.9408@gmail.com (Carlos David Morales Peña), diogo@cpdee.ufmg.br (Diogo Batista de Oliveira), elson@cpdee.ufmg.br (Elson José da Silva), mauricio.weber@gmail.com (Maurício Weber Benjó da Silva) 


\section{Introduction}

Nowadays, RFID (Radio Frequency Identification) systems are widely used in wireless communication to track and identify objects of a precise and reliable way. In medical and industrial area is required the constant supervision and care of instruments, equipment, inventory in a reduced time, with the aim to supply an optimum and innovative service. Additionally, the control access to restricted areas implies a reliable security system which may include the use of bracelets and cards with RFID tags.

The RFID system comprises a reader, tag, computer host and communication network. Its operating principle is based on sending a modulated RF signal by a reader, to feed and interrogate the passive tag. The tag is composed of a tag antenna and a chip IC (Integrated circuit), which modifies its impedance and produces a backscattering signal [1]. In addition, the RFID signal can be transmitted in several frequency bands. Here, 902-928 MHz band has been mostly used in Ultra High Frequencies (UHF) [2].

One of the most important challenges when designing RFID tags is the antenna size-performance ratio and the undesired interaction with surrounded bodies [1, 3]. RFID system is generally sensitive to noises generated by signal interference and multi-path effect, which mainly affect the backscattering process [4]. Thus, the development of tags mountable on platforms with different shapes and materials makes it difficult to accomplish directional radiation patterns, large reading ranges, and low sensitivity in terms of frequency band and gain.

The antenna design to label metallic objects have several electromagnetic constraints to be considered. It is well-known that conductive surfaces generates an image current which can cancel the antenna current due to the phase difference of $180^{\circ}$ between them. This can affect the input impedance, resonant frequency, and antenna gain [5]. In addition to this difficulty, the effect of the antenna curvature plays an important role in the antenna performance. Here, the resonance frequency and antenna gain are two sensitive parameters affected by the antenna bending [6]. Then, the need for performing RFID tags considering the previous limitations is a goal of paramount importance for designers.

Flexible slots and dipole antennas made of a polymer-ceramic composite substrate is mainly suggested for RIFD tag design for tracking small items [1]. Here, most of the published papers propose the development of flexible substrates using polymers in order to increase the substrate permittivity 
$[1,7]$. However, the manufacturing of these kind of substrates is developed home-made or in non-commercial laboratories, making difficult its acquisition. Additionally, the manufacturing process does not keep a high quality standard to guarantee the good performance of the substrate for high frequencies and its electrical parameters are not provided with accuracy. These factors represent an important limitation for the antenna design.

This paper proposes a methodology to design a RFID tag mounted on curved metallic surfaces. The starting point is the tag antenna originally proposed [7]. As main contribution of this work, we use a flexible commercial substrate to design the tag antenna. The resulting prototype is an ultra-slim and electrically small antenna, which is an advantage over the original design [7], when used in applications involving small objects.

The design parameters of the antenna are modified using a Genetic Algorithm to optimize the antenna performances. The optimization process is based on the manipulation of the parameters which involve the T-matching network and slots. Also, the effects of the antenna curvature are analyzed based on the cylindrical cavity model in order to understand the impact on the resonance frequency and antenna efficiency when mounted on metallic and polyethylene terephthalate (PET) bottles.

\section{Antenna design}

The selected antenna is a slot dipole, as shown in Figure 1. The antenna has a dimension of $13 \mathrm{~mm} \times 28 \mathrm{~mm}$. The tag is equipped with a chip of reference NXP G2iL [8]. It has a complex impedance $23-224 \mathrm{j} \Omega$ at central frequency of $915 \mathrm{MHz}$. Besides, the chip has a sensitivity $P_{I C}=-18 \mathrm{dBm}$. The flexible substrate used is Rogers Ultralam 3850HT [9]. The electric properties of the selected substrate were calculated considering the roughness of the double cladding laminated $[10,11]$. Here, $\epsilon_{r}=2.6$ and $\tan \delta=0.03$ is

obtained. Additionally, the antenna substrate has a cylindrical geometry with radius $=16 \mathrm{~mm}$ and thickness of $0.35 \mathrm{~mm}$.

\subsection{Antenna structure}

The tag is formed by two main combinations that involve methods to conjugate the impedance matching between the chip and tag antenna. These elements are T-matching and shaped slots. T-matching is made of a slot which guide the current in a same direction, modifying the inductive impedance of the antenna. Thus, this network is an interesting technique which allows 


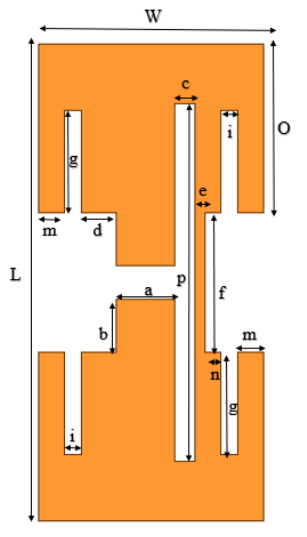

(a)

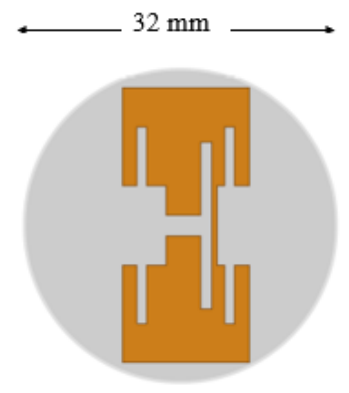

(b)

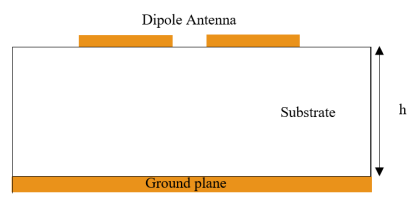

(c)

Figure 1: Proposed antenna structure seen from: a) front view, b) front view with substrate, c) side view.

small antennas accomplish a good performance on impedance matching and gain.[12].

The $p$ parameter is a sensible variable in the T-matching network. Figure 2 and 3 illustrate the power reflection coefficient and normalized gain of the tag antenna for three different cases: when the antenna is isolated, mounted on a square metallic plate and mounted on a metallic cylinder. An increment of $p$ produces an increase of the antenna inductance, and therefore, the reduction of the resonance frequency. Additionally, the return loss of the tag antenna decreases for high values of $p$. Regarding the antenna gain, it is observed that antenna pattern is different for the three different cases. The gain depends on the structure where the antenna is mounted. Furthermore, a variation of $p$ produces a small impact on the radiation pattern for some values of $\theta$.

\subsection{Selection of substrate}

According to the disadvantages using a non-commercial substrate, a selection criteria was defined based on the following items: acquisition in the market, good performance in high frequencies, a high dielectric constant, and 


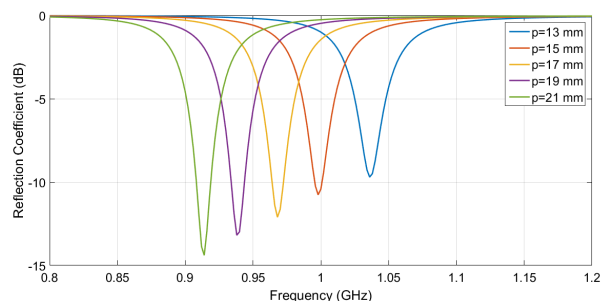

(a)

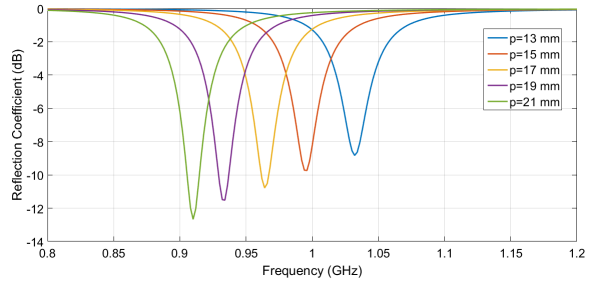

(b)

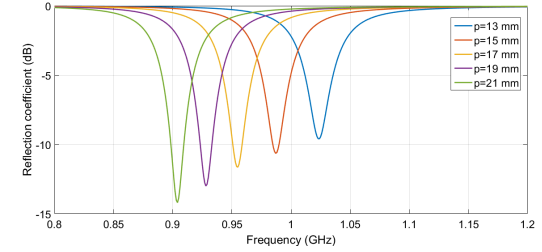

(c)

Figure 2: Reflection coefficient of the tag for a variation of the parameter $\mathrm{p}$ related to the T-match network when the antenna is (a) isolated, (b) mounted on a metallic plate of 125 $\mathrm{mm} \times 125 \mathrm{~mm}$, and (c) mounted on a metallic cylinder with radius of $20 \mathrm{~mm}$.

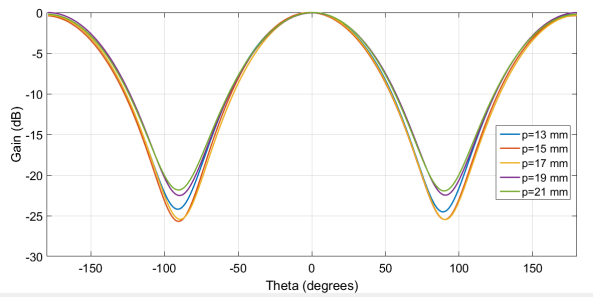

(a)

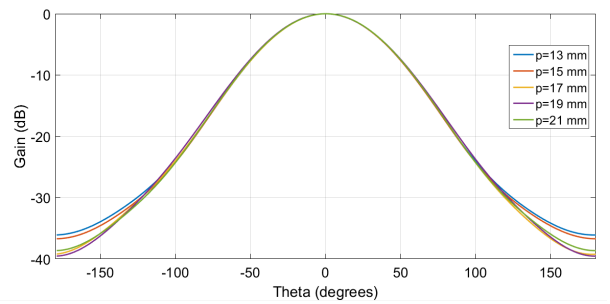

(b)

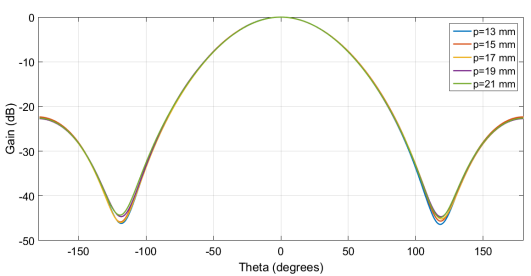

(c)

Figure 3: Normalized gain of the tag for a variation of the parameter $\mathrm{p}$ related to the T-match network when the antenna is (a) isolated, (b) mounted on a metallic plate of 125 $\mathrm{mm} \times 125 \mathrm{~mm}$, and (c) mounted on a metallic cylinder with radius of $20 \mathrm{~mm}$.

a low dielectric loss. Thus, based on the previous criteria, the Rogers Ultralam 3850 HT substrate was selected. Furthermore, the substrate thickness 
was chosen based on the antenna performance for the selected application. Hence, simulations were run to determine the proper thickness. Here, parameters as: resonance frequency, radiation efficiency and antenna gain, differ depending on the selected thickness. Based on the results, as the substrate thickness increases, the resonance frequency, radiation efficiency and antenna gain increase, as depicted in Figure 4.

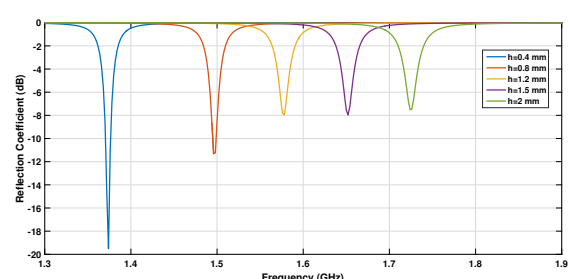

(a)

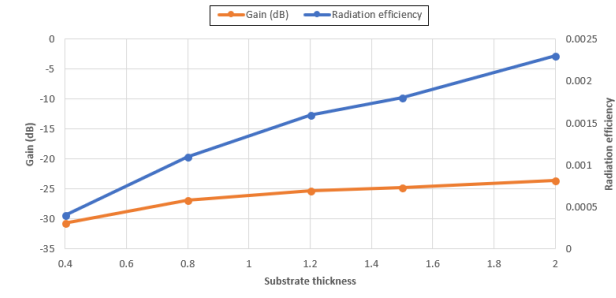

(b)

Figure 4: (a) Reflection coefficient of the tag antenna for a variation of the substrate thickness, and (b) variation of antenna gain and radiation efficiency in function of the substrate thickness.

Moreover, according to the analysis of the cavity model without losses for microstrip antennas, the increase of the substrate thickness generates the reduction of the resonance frequency $\left(f_{r 0}\right)[13]$. This is due to the increment of the effective length, produced for the fringing effects in the antenna. Nevertheless, the variation of the stored energy in the cavity produces a dissipated power, represented in function of dielectric losses. Then, studying the cavity model with losses, the increment of the quality factor $(Q)$, which occurs for an increase of the substrate thickness, produces an increment in the resonance frequency $\left(f_{r}\right)$ of the antenna, as expressed in the following equation

$$
f_{r}=f_{r 0}\left(1-\frac{1}{2 Q}\right)
$$

However, the radiation fields in microstrip antennas depends on the substrate thickness. An increase of the substrate thickness produces an increment of the electric field, leading to increase the radiation power of the antenna. Also, the increase of the substrate thickness allows the antenna to decrease the dissipated power. Consequently, the radiation efficiency of the antenna enhances. In such a way, a substrate thickness with an optimal balance among the mentioned electrical parameters was considered to achieve 
good antenna performance. Here, two laminates of ULTRALAM 3850HT (each laminate with thickness of $0.175 \mathrm{~mm}$ ) were used to create a single substrate with thickness of $0.35 \mathrm{~mm}$.

\section{Analysis of the antenna curvature}

The analysis of small antennas mounted on cylindrical surfaces comprises the analysis of the cylindrical cavity model and effect of the antenna curvature over the resonance frequency and radiation field solution. The substrate is considered as a cavity with electric walls on the top and bottom (conducting antenna and grounded cylindrical surface) and magnetic walls on the side (dielectric cavity), to obtain the fields and the associated modal resonance frequencies. Moreover, assuming that $h \ll \lambda$ and $h \ll a$, only TM modes radiate into the cylindrical cavity $[6,14]$. Then, using cylindrical coordinates, the source-free electric field satisfies the wave equation as follows

$$
\frac{1}{\rho} \frac{\partial \rho E_{\phi}}{\partial \rho \partial \phi}-\frac{1}{\rho^{2}} \frac{\partial^{2} E_{\rho}}{\partial \phi^{2}}-\frac{\partial^{2} E_{\rho}}{\partial z^{2}}+\frac{\partial^{2} E_{z}}{\partial z \partial \rho}-k^{2} E_{\rho}=0
$$

Where:

$$
k^{2}=\left(\frac{m \pi}{2 \theta(a+h)}^{2}\right)+\left(\frac{n \pi}{L}\right)^{2}
$$

Afterwards, it is possible to assume the electric field is assumed to have only a component. Then,

$$
E_{\rho}=-\frac{j}{\omega \mu \epsilon} k_{\rho}\left(\frac{n \pi}{L}\right) A_{m n} R_{v}^{\prime}\left(k_{\rho} \rho\right) \sin \left(\frac{n \pi}{L} z\right) \cos \left(\frac{m \pi}{2 \theta} \phi\right)
$$

where $k$ is the wave number, $\omega$ is the angular frequency, $a$ is the radius of the cylinder, $\epsilon$ is the substrate permittivity, $\mu$ is the substrate permeability, $L$ is the dimension of the straight edge of the tag antenna, $h$ is the substrate thickness, $\theta$ is the bend angle, and the curved edge is expressed by $2(a+h) \theta$.

$$
k_{\rho}=\frac{m \pi}{2 \theta(a+h)}
$$

Finally, the resonance frequency can be written as:

$$
f_{r}=\frac{1}{2 \epsilon \mu} \sqrt{\left(\frac{m \pi}{2 \theta(a+h)}\right)^{2}+\left(\frac{n \pi}{L}\right)^{2}}
$$


Equation (4) displays the relation of the electric field stored in the cavity, in function of the antenna curvature. Then, an increase of the antenna curvature produces the decrease of the electric field in the cavity, which it affects the radiation efficiency of the antenna. Moreover, based on (6), it is possible to affirm that the antenna curvature affects the resonance frequency of the tag antenna. Then, an increment of the angle $\theta$ generates a slight reduction of the resonance frequency.

The following equation represents the average dissipated power

$$
<P_{d}>=\frac{\omega}{2}|\mathbf{E}|^{2} \epsilon^{\prime \prime}
$$

where $\epsilon^{\prime \prime}$ is the imaginary part of the complex permittivity and it models the dielectric losses of the cavity, which it depends only of the frequency.

According to (7), a decrease of the electric field in the cavity produces the reduction of the dissipated power in the cavity. Moreover, the radiated fields of the antenna depends on the radius of the cylinder where the antenna is mounted. An decrease of the radius of cylinder generates an increase of the electric field provided by the antenna [6]. Consequently, the radiated power and the radiation efficiency increase, improving the antenna performance.

These concepts explain how the antenna curvature affects its electromagnetic properties, and why is important to design and optimize the tag antenna for a predefined structure in order to obtain maximum read range. Therefore, the optimization process applied directly on the surface to be labeled is suggested to achieve the best antenna performance.

\section{Optimization process}

For improving the antenna performance, the main challenge in RFID systems is to maximize the tag read range. Hence, to satisfy this goal, a maximum power transfer between antenna and chip is needed for enhancing the antenna gain. The goal is to accomplish a good transmission efficiency which allows to generate the backscattering signal by the tag.

In order to evaluate the antenna performance, it is useful to determine how well the antenna and chip are matched. The transmission coefficient is an indicator which defines the state of the impedance matching and is given by

$$
\tau=\frac{4 R_{a} R_{\text {chip }}}{\left|Z_{a}+Z_{\text {chip }}\right|^{2}}
$$


Where $Z_{a}=R_{a}+\mathrm{j} X_{a}$ and $Z_{c h i p}=R_{\text {chip }}+\mathrm{j} X_{\text {chip }}$ are the complex antenna and microchip input impedance, respectively.

Subsequently, the read range can be defined as the maximum distance at which the reader can detect the tag antenna. The read range can be expressed by using the Friis equation

$$
d_{\text {tag }}=\frac{\lambda}{4 \pi} \sqrt{\frac{G_{t a g} \tau E I R P}{P_{I C}}}
$$

Where $P_{I C}$ is the chip sensitivity, $G_{t a g}$ is the tag antenna gain, $\tau$ is the transmission coefficient and EIRP represents the equivalent isotropic radiated power, defined as $4 \mathrm{~W}$ at $915 \mathrm{MHz}$, according to the normative [15]. Also, a polarization matching between the reader and tag is assumed perfect. Then, the polarization efficiency is 1 .

For achieving a good antenna performance, an optimization process on the geometry of the antenna was applied, using a Genetic Algorithm (GA) by a MATLAB's toolbox. GA method is a stochastic search algorithm with good characteristics of local search and generation of diversity $[16,17]$. The objective of the optimization is to maximize the read range of the tag at central frequency of $915 \mathrm{MHz}$. In the optimization process, firstly, an parametric analysis was developed, including all parameters of the tag, to determine which antenna parameters are the most sensitive to improve the impedance matching and the antenna gain. In this sense, 6 parameters $(a, c, f, g, n$, and $p$, according to Figure 1), were selected in a specific search range to maximize the antenna performance.

Moreover, in the GA optimization, a population size of 25 individuals and 10 generations were defined, where the population is submitted to selection, recombination, and mutation operators to find the optimum values. Here, were chosen the selection function roulette and a recombination fraction of 0.8. The algorithm initializes the first generation randomly and operates until the last generation where it finds the best antenna parameters to maximize its read range. The selection criteria on the number of individuals and generations was based on the analysis of convergence of the objective function and the execution time of the algorithm. Moreover, in the algorithm, two individuals were selected as elite population, representing almost $10 \%$ of the population, for saving a record of the best results for each generation. 


\section{Performance of the tag antenna on metallic surfaces}

The tag antenna is simulated in HFSS v.15 to evaluate its electromagnetic properties. The conductor of the tag antenna was modeled as copper and the substrate as a dielectric (see section 2). The tag was modeled as an integration of a dipole antenna and a lumped port with complex impedance to model the chip IC. A central frequency of $915 \mathrm{MHz}$ was selected to compute the far field electromagnetic solution of the tag antenna. Moreover, the metallic cylinders were modeled as copper surface with a height of $120 \mathrm{~mm}$.

In this section, two optimizations are presented. The first optimization consists in maximizing the read range of the tag antenna directly on metallic cylinders. The second optimization of the tag antenna was developed on a copper plate. Here, its electrical performance is analyzed considering the effect of the antenna bending, when the antenna is mounted on copper cylinders.

\subsection{Optimization on copper cylinder}

Table 1 shows the parameters of the optimized antenna mounted on a metallic cylinder of radius $=20 \mathrm{~mm}$, using a Genetic Algorithm.

Table 1: Parameters and dimension of the tag antenna mounted on a copper cylinder using genetic algorithm.

\begin{tabular}{cccccc}
\hline Parameter & $\begin{array}{c}\text { Length } \\
(\mathbf{m m})\end{array}$ & Parameter & $\begin{array}{c}\text { Length } \\
(\mathbf{m m})\end{array}$ & Parameter & $\begin{array}{c}\text { Length } \\
(\mathbf{m m})\end{array}$ \\
\hline $\mathrm{a}$ & 3.35 & $\mathrm{f}$ & 8.2 & $\mathrm{n}$ & 0.9 \\
$\mathrm{~b}$ & 2 & $\mathrm{~g}$ & 6 & $\mathrm{O}$ & 9.9 \\
$\mathrm{c}$ & 1.2 & $\mathrm{~h}$ & 0.35 & $\mathrm{p}$ & 21 \\
$\mathrm{~d}$ & 2 & $\mathrm{i}$ & 1 & $\mathrm{~L}$ & 28 \\
$\mathrm{e}$ & 0.55 & $\mathrm{~m}$ & 1.5 & $\mathrm{~W}$ & 13 \\
\hline
\end{tabular}

Figure 5 shows the (a) 2D and (b) 3D radiation patterns of the tag antenna over a metallic cylinder with radius $=20 \mathrm{~mm}$, placed at $y$-axis. As observed, the maximum antenna gain is $-13.37 \mathrm{dBi}$ at $\theta=0^{\circ}$.

The effective area of the antenna is wider for an increase of the antenna curvature. Thereby, the radiation pattern presents a secondary lobe that improves the coverage area of the tag. This behavior is more noticeable for an increase of the antenna bending. 


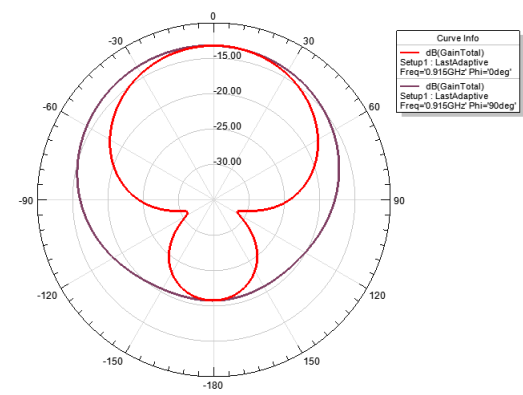

(a)

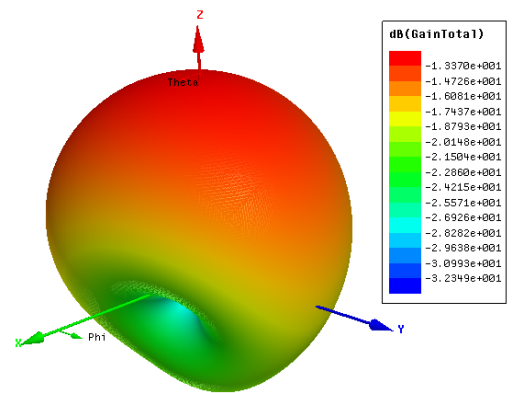

(b)

Figure 5: 2D and 3D radiation pattern of the tag antenna on metallic cylinder with radius $=20 \mathrm{~mm}$.

Figure 6 (a) illustrates the electrical behavior of the antenna in terms of radiation efficiency and gain with a variation of radius of the cylinder. The antenna directivity decreases with an increment of the curvature, due to part of energy is radiated in the back side of the antenna. Moreover, its radiation efficiency enhances as the antenna curvature increases, and therefore, the antenna gain and read range are improved, as depicted in Figure 6 (b). These results follow the analyzed hypothesis of the cavity model for curved antennas.

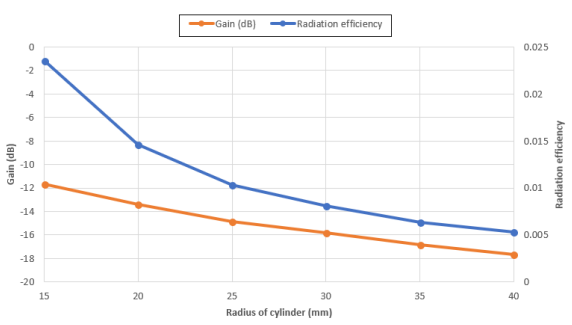

(a)

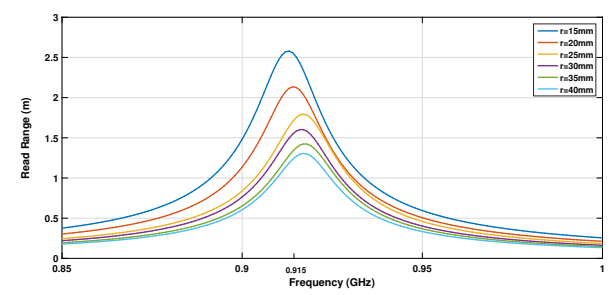

(b)

Figure 6: (a) Transmission efficiency and gain of the tag antenna over cylindrical metallic objects, and (b)read range of the tag antenna over cylindrical metallic objects with different radii.

Table 2 shows the transmission coefficient, gain and read range of the optimized tag antenna on cylindrical metallic objects with different radii. According to the results, the resonance frequency of the tag antenna decreases for an increment of the antenna curvature. Also, the read range increases with the antenna curvature, improving the tag performance. 
Table 2: Simulated results of the tag antenna wrapped on cylindrical metallic objects with different radii at $915 \mathrm{MHZ}$.

\begin{tabular}{ccccccc}
\hline Parameter & $\mathbf{1 5 m m}$ & $\mathbf{2 0 m m}$ & $\mathbf{2 5 m m}$ & $\mathbf{3 0 m m}$ & $\mathbf{3 5 m m}$ & $\mathbf{4 0 m m}$ \\
\hline $\begin{array}{c}\text { Transmission } \\
\text { Coefficient }\end{array}$ & 0.54 & 0.57 & 0.54 & 0.56 & 0.54 & 0.54 \\
$\begin{array}{c}\text { Gain }(\mathrm{dBi}) \\
\quad-11.68\end{array}$ & -13.37 & -14.84 & -15.84 & -16.85 & -17.63 \\
$\quad \begin{array}{r}\text { Read } \\
\text { Range }(\mathrm{m})\end{array}$ & 2.51 & 2.13 & 1.75 & 1.58 & 1.38 & 1.27 \\
\hline
\end{tabular}

Additionally, an evaluation of the antenna performance on a copper cylinder with radius $=80 \mathrm{~mm}$ was done. It is observed that the antenna read range decreases considerably to $0.63 \mathrm{~m}$ at $915 \mathrm{MHz}$, similar coverage than the antenna performance on a copper plate with $125 \mathrm{~mm}$ x $125 \mathrm{~mm}$. Therefore, a new optimization was developed, using the genetic algorithm for the antenna mounted on cylinder copper of radius $=40 \mathrm{~mm}$. The optimized antenna achieved a read range about $1.3 \mathrm{~m}$ at $915 \mathrm{MHz}$. Despite maximizing the antenna read range on cylinders with higher radii, the antenna tends to decrease its curvature and behaving like a planar surface, decreasing its read range.

\subsection{Optimization on copper plate}

The selected dipole antenna does not present a good performance on planar metallic objects since the read range is lower than $1 \mathrm{~m}$. Therefore, the tag antenna performance is considered reliable only when it is wrapped on curved volumes, as consequence the effect of antenna bending which it improves the read range. Moreover, the use of the Genetic Algorithm directly on the copper cylinder allows the antenna performance to be improved around of $37 \%$ than the antenna optimization on a flat surface, when the applicability of the RFID tag is on metallic curved surface of radius $=20 \mathrm{~mm}$. Noticeably, the application of the Genetic Algorithm directly on the copper cylinder with respect to a planar surface is very important to achieve an optimum maximization of the read range in the desired frequency, as shown in Figure 7 .

Moreover, in order to maximize the antenna read range, it is important not only consider a good impedance matching; the antenna gain also plays an essential role. Figure 8 illustrates the read range variations of the dipole antenna for the three cases analyzed: an antenna 1 (optimized antenna on copper plate of $125 \mathrm{~mm}$ x $125 \mathrm{~mm}$ and mounted on copper plates), an antenna 


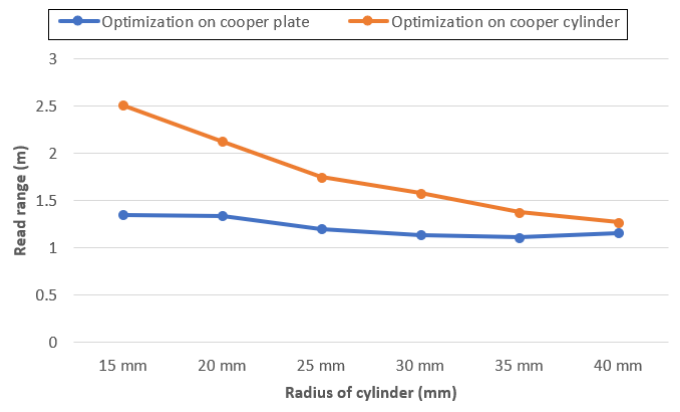

Figure 7: Read range of the tag antenna mounted on copper cylinders when it is optimized on metallic curved and flat surfaces.

2 (optimized antenna on copper plate of $125 \mathrm{~mm}$ x $125 \mathrm{~mm}$ and mounted on copper cylinders) and an antenna 3 (optimized antenna on copper cylinder of radius $=20 \mathrm{~mm}$ and mounted on copper cylinders). According to the figure, tags mounted on a cylindrical surface, characterized for its high gain and limited impedance matching, have a better read range than tags wrapped on a flat surface, with properties of low gain and high impedance matching. Moreover, using the optimization directly on the surface to be labeled (antenna 3) represents the best methodology to obtain a maximum coverage and a high antenna performance.

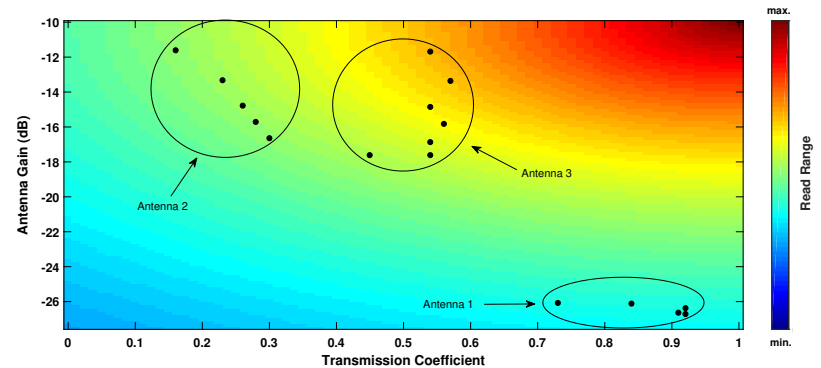

Figure 8: Variation of read range in function of power transmission coefficient and gain. The red color represents a maximum read range and the blue color a minimum coverage.

\section{Performance of the tag antenna on PET surfaces with and with- out liquids}

In this section, the antenna performance on PET bottles is analyzed, with a thickness of $0.8 \mathrm{~mm}$ and the following electrical properties: dielec- 
tric constant of 2.8 and dielectric loss tangent of 0.003 . Here, the antenna performance on PET bottles with and without liquid content is analyzed. Table 3 shows the antenna parameters, based on the results obtained using the Genetic Algorithm, which maximizes the read range of the tag antenna for a PET cylinder of radius $=20 \mathrm{~mm}$.

Table 3: Parameters and dimension of the tag antenna mounted on a PET cylinder using genetic algorithm.

\begin{tabular}{cccccc}
\hline Parameter & $\begin{array}{c}\text { Length } \\
(\mathbf{m m})\end{array}$ & Parameter & $\begin{array}{c}\text { Length } \\
(\mathbf{m m})\end{array}$ & Parameter & $\begin{array}{c}\text { Length } \\
(\mathbf{m m})\end{array}$ \\
\hline $\mathrm{a}$ & 3.3 & $\mathrm{f}$ & 8 & $\mathrm{n}$ & 0.8 \\
$\mathrm{~b}$ & 2 & $\mathrm{~g}$ & 6 & $\mathrm{O}$ & 10 \\
$\mathrm{c}$ & 1.2 & $\mathrm{~h}$ & 0.35 & $\mathrm{p}$ & 17.5 \\
$\mathrm{~d}$ & 2 & $\mathrm{i}$ & 1 & $\mathrm{~L}$ & 28 \\
$\mathrm{e}$ & 0.7 & $\mathrm{~m}$ & 1.5 & $\mathrm{~W}$ & 13 \\
\hline
\end{tabular}

\subsection{Tag antenna on empty PET bottle}

The radiation pattern of the tag antenna on an empty plastic cylinder with radius $=20 \mathrm{~mm}$ is shown in Figure 9. The antenna gain is $-17.96 \mathrm{dBi}$ and its radiation pattern presents a front-back ratio close to unity. In addition, the radiation efficiency of the antenna is lower than those obtained for the antenna wrapped on metallic cylinders.

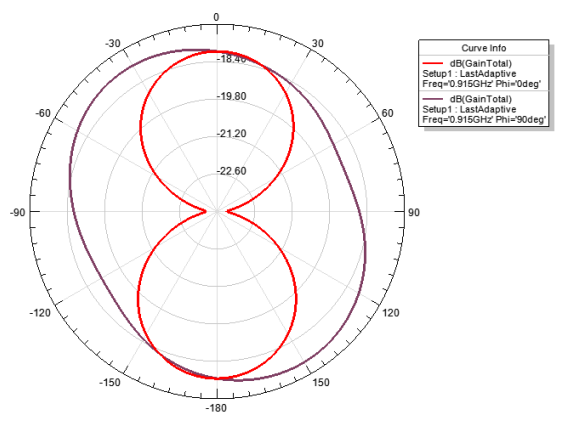

Figure 9: Gain of the proposed tag antenna on empty $P E T$ bottle with radius $=20 \mathrm{~mm}$ in the XZ plane $\left(\phi=0^{\circ}\right)$ and $\mathrm{YZ}$ plane $\left(\phi=90^{\circ}\right)$.

The antenna has a lower directivity on plastic cylinders than those of metallic cylinders, as consequence of absorbent properties of PET (good dielectric). On the other hand, the increase of the antenna curvature improves 
the radiation efficiency, enhancing the gain and reducing the resonance frequency. Table 4 show the gain and the read range of the tag antenna for different empty PET cylinders. As observed, the proposed antenna operates at the central frequency of $915 \mathrm{MHz}$ with about of one meter of coverage.

Table 4: Simulated results of the tag antenna on empty PET bottles with different radii at $915 \mathrm{MHz}$

\begin{tabular}{|c|c|c|c|c|c|c|}
\hline Parameter & $15 \mathrm{~mm}$ & $20 \mathrm{~mm}$ & $25 \mathrm{~mm}$ & $30 \mathrm{~mm}$ & $35 \mathrm{~mm}$ & $40 \mathrm{~mm}$ \\
\hline $\begin{array}{c}\text { Transmission } \\
\text { Coefficient }\end{array}$ & 0.29 & 0.35 & 0.34 & 0.30 & 0.30 & 0.32 \\
\hline Gain (dBi) & -17.55 & -17.96 & -18.32 & -18.46 & -18.6 & -18.73 \\
\hline $\begin{array}{c}\text { Read } \\
\text { Range }(\mathrm{m})\end{array}$ & 0.93 & 0.98 & 0.92 & 0.85 & 0.84 & 0.86 \\
\hline
\end{tabular}

\subsection{Tag antenna on PET bottle with liquid}

The RFID tag antenna presents a variation of its electromagnetic properties when different kind of liquids inside the plastic cylinders are added. Basically, this variation is produced by the adding a new dielectric with the PET volume, creating a multilayer dielectric with a higher effective permittivity. Table 5 and Figure 10 show the influence of the liquid content in a plastic cylinder with radius $=20 \mathrm{~mm}$ on the tag performance. The analysis was developed for five liquids: water, glycerin, ethanol, acetone, and olive oil, which have a dielectric constant of 81, 47, 24.3, 20.7 and 3.1; and dielectric loss tangent of $0.157,0.2,0.25,0.25$ and 0.04 , respectively. As observed for all liquids, the tag antenna operates at the required frequency band. Besides, the liquids with higher dielectric constants produce a high antenna gain and read range.

Table 5: Simulated results of the tag antenna on a PET bottle of radius=20 $\mathrm{mm}$ with different liquid contents at $915 \mathrm{MHz}$.

\begin{tabular}{cccccc}
\hline Parameter & Water & Glycerin & Ethanol & Acetone & Oliver Oil \\
\hline $\begin{array}{c}\text { Transmission } \\
\text { Coefficient }\end{array}$ & 0.36 & 0.36 & 0.35 & 0.34 & 0.40 \\
$\begin{array}{c}\text { Gain (dBi) } \\
\text { Read }\end{array}$ & -10.54 & -12.76 & -16.83 & -17.98 & -18.84 \\
Range $(\mathrm{m})$ & 2.34 & 1.81 & 1.12 & 0.96 & 0.95 \\
\hline
\end{tabular}




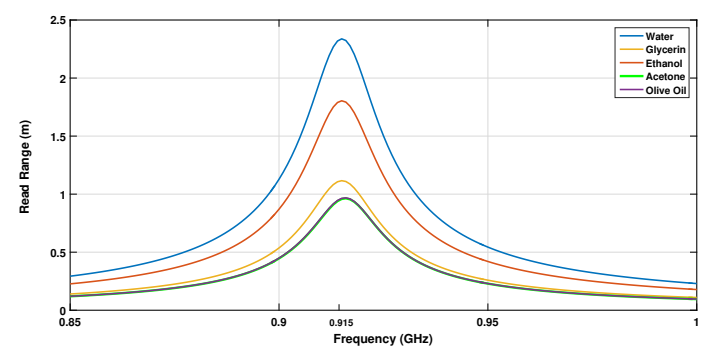

Figure 10: Read range of the tag antenna on PET bottle of radius=20 $\mathrm{mm}$ with different liquid contents.

The radiation efficiency of the RFID antenna over a PET bottle with liquid content is low because of the absorption of electromagnetic energy by the multilayer dielectric. Furthermore, the tag antenna on a PET bottle with liquid content can potentially increase the antenna impedance, leading the improvement of the radiation efficiency [3].

Moreover, the PET bottle with a liquid of high permittivity, act as a conductor plane, reflecting the incident signals provided from the reader. Thus, the antenna directivity improves. In addition, as shown in (7), the dissipated power depends on the electric field and the dielectric loss tangent of the dielectric cavity. Then, for low values of permittivity, the dielectric layer formed by the PET bottle and the liquid absorb part of the incident energy, increasing the dissipated power, which leads to obtain a low radiation efficiency, as illustrated in Figure 11. Also, the current density distribution on the PET cylinder increases as the liquid permittivity rises.

However, for olive oil, liquid with the lowest permittivity, the radiation efficiency is higher than acetone and ethanol. This is due to its dielectric loss tangent, which is very low compared with the other liquids. Therefore, the PET bottle with olive oil allows the antenna to dissipate less energy than acetone and ethanol.

On the other hand, the high dielectric constant of water allows to reduce the secondary lobes or nulls in the radiation pattern of the tag antenna. For these reason, the radiation pattern tends to be more directive and the read range of the tag enhances, when the dielectric constant of the liquid increases. 


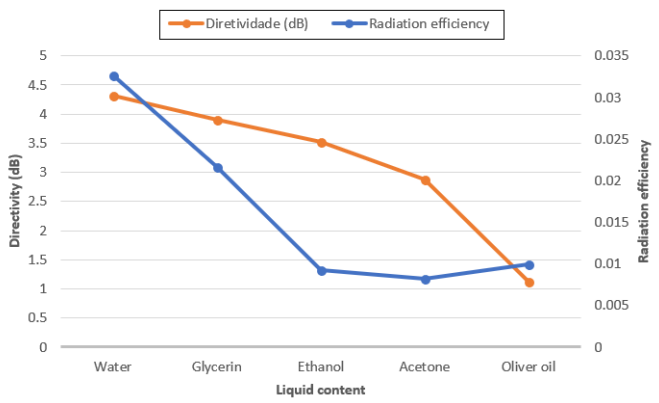

Figure 11: Directivity and radiation efficiency of the tag antenna over PET bottle of radius $=20 \mathrm{~mm}$ with different liquid contents.

\section{Prototype}

The parameters of the antenna built were shown in Table 1. Here, antenna measurements on a copper cylinder with radius $=20 \mathrm{~mm}$ were developed. The differential S-parameter method was used for developing measurements of reflection coefficient of the tag antenna [18]. Here, Rohde \& Schwarz ZVL-6 Vector Network Analyzer was employed to measure the matrix S-parameter.

Figure 12 (a) illustrates the built prototype, and 12 (b) shows the simulated and measured reflection coefficient of the tag antenna mounted on a copper cylinder with a radius of $20 \mathrm{~mm}$. The antenna is tuned in the 902$928 \mathrm{MHz}$ frequency band with a measured reflection coefficient of $-6.8 \mathrm{~dB}$, showing a good agreement with simulations.

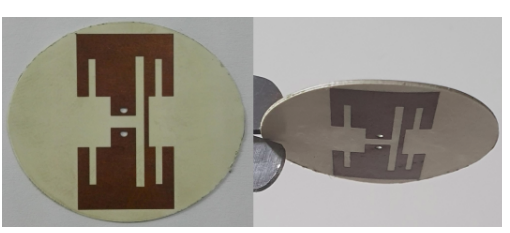

(a)

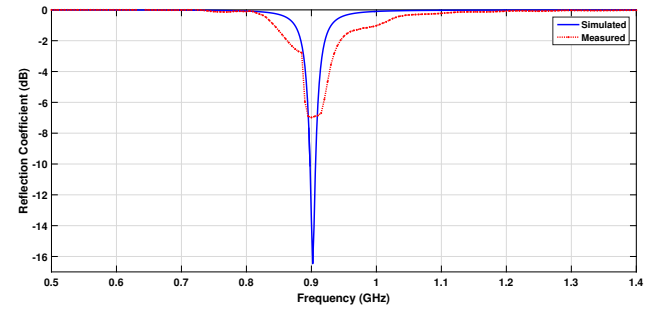

(b)

Figure 12: a) Prototype, and b) measured vs. simulated reflection coefficient of the proposed tag antenna wrapped on a copper cylinder of radius $=20 \mathrm{~mm}$.

Finally, Table 6 shows a comparison of the proposed antenna mounted on metallic and PET cylinders with some antenna designs published in the literature. The comparison relies on the antenna performances at $915 \mathrm{MHz}$. 
Table 6: Comparison of the proposed tag antenna with different designs.

\begin{tabular}{|l|c|c|c|c|c|}
\hline Ref. & Proposed & {$[7]$} & {$[19]$} & {$[20]$} & {$[3]$} \\
\hline Cylinder & $\begin{array}{c}\text { Metal. } \\
\text { Diameter } \\
=50 \mathrm{~mm}\end{array}$ & $\begin{array}{c}\text { Metal. } \\
\text { Diameter } \\
=50 \mathrm{~mm}\end{array}$ & $\begin{array}{c}\text { Metal. } \\
\text { Diameter } \\
=40 \mathrm{~mm}\end{array}$ & Metal. & $\begin{array}{c}\text { PET. } \\
\text { Diameter } \\
=64 \mathrm{~mm}\end{array}$ \\
\hline $\begin{array}{l}\text { Antenna } \\
\text { volume }\left(\mathrm{mm}^{3}\right)\end{array}$ & 281.5 & 1206.4 & 5000 & 1350 & 761.7 \\
\hline Gain $(\mathrm{dBi})$ & -14.8 & -10.3 & - & -8.8 & -10.8 \\
\hline Read range $(\mathrm{m})$ & 1.75 & 2.8 & 0.95 & 1.8 & 2.5 \\
\hline
\end{tabular}

As observed, the proposed antenna wrapped on metallic and PET cylinders has a good performance in terms of gain and read range, considering that it has the smallest volume among the designs presented in the table.

\section{Conclusions}

A ultra slim and electrically small passive UHF RFID tag antenna has been designed. The antenna can be wrapped on cylindrical metallic surfaces with radii lower than $40 \mathrm{~mm}$, achieving read ranges above one meter. Furthermore, a maximum read range of $2.5 \mathrm{~m}$ has been accomplished when the antenna is mounted on a cylinder with radius of $15 \mathrm{~mm}$. Given these antenna performances, the proposed tag antenna can be used in real applications to track small items, such as warehouse management for detecting hardware tools, kitchenware, tin cans, and spray bottles; industrial and medical field for tracking curved tools and monitoring equipment calibration; and finally, access control systems equipped with RFID cards and bracelets.

The RFID antenna has been optimized by using a Genetic Algorithm in order to maximize its read range. Here, read ranges above one meter have been achieved when mounted over cylindrical metallic objects. On the other hand, the tag antenna is considered unsuitable to label flat surfaces and PET bottles with liquid content of low permittivity.

The proposed RFID tag antenna can be wrapped on empty PET bottles and with liquid content. The results show that the proposed antenna made of a flexible commercial substrate can operate with a read range of above one meter. Moreover, the electrical properties of the liquid content can improve the antenna performances due to the capability for working as conductor ground, leading to enhance the radiation efficiency of the tag antenna. 


\section{References}

[1] L. Ukkonen, Y. Rahmat-samii, Advances in Antenna Designs for UHF RFID Tags Mountable on Conductive Items (2014). doi:10.1109/MAP.2014.6821761.

[2] J.-S. Sun, C.-H. Wu, A broadband circularly polarized antenna of square-ring patch for UHF RFID reader applications, AEU-International Journal of Electronics and Communications 85 (2018) 84-90.

[3] T. Bjorninen, A. Z. Elsherbeni, L. Ukkonen, Low-profile conformal UHF RFID tag antenna for integration with water bottles, IEEE Antennas and Wireless Propagation Letters 10 (2011) 1147-1150.

[4] L. Xie, Y. Yin, A. V. Vasilakos, S. Lu, Managing RFID data: challenges, opportunities and solutions, IEEE communications surveys \& tutorials 16 (3) (2014) 1294-1311.

[5] A. A. Babar, V. A. Bhagavati, L. Ukkonen, A. Z. Elsherbeni, P. Kallio, L. Syd, Performance of High-Permittivity Ceramic-Polymer Composite as a Substrate for UHF RFID Tag Antennas, Internation Journal of Antennas and Propagation 2012 (2012). doi:10.1155/2012/905409.

[6] C. M. Krowne, Cylindrical-Rectangular Microstrip Antenna, IEEE Transactions on Antennas and Propagation 31 (1) (1983) 194-199. doi:10.1109/TAP.1983.1143000.

[7] A. Babar, P. Kallio, L. Ukkonen, Small and Flexible Metal Mountable Passive UHF RFID Tag on High-Dielectric Polymer-Ceramic Composite Substrate, IEEE antennas and wireless propagation letters 11 (2012) 1319-1322. doi:10.1109/LAWP.2012.2227291.

[8] NXP Semiconductor, SL3S1203_1213 UCODE G2iL and G2iL+ IC Chip (32) (2014).

[9] Rogers Corporation, Datasheet Ultralam @ 3850ht 1-2.

[10] X. Guo, D. R. Jackson, J. Chen, An analysis of copper surface roughness effects on signal propagation in PCB traces, Texas Symp. on Wireless and Microw. Circuits Syst. (WMCS) (2013). 
[11] J. Reynolds, P. LaFrace, Effect of conductor profile on the insertion loss, phase constant, and dispersion in thin high frequency transmission lines, Tech. rep., Rogers Corporation (2010).

[12] Y. J. Zhang, D. Wang, M. S. Tong, An adjustable quarter-wavelength meandered dipole antenna with slotted ground for metallically and airily mounted RFID tag, IEEE Transactions on Antennas and Propagation 65 (6) (2017) 2890-2898.

[13] C. Balanis, Antenna Theory: Analysis and Design, 3rd Edition, no. 3, 2005.

[14] K.-M. Luk, K.-F. Lee, J. Dahele, Analysis of the Cylindrical-Rectangular Patch Antenna, IEEE Transactions on Antennas and Propagation (1989).

[15] M. Bolic, I. Stojmenovic, RFID systems Research Trends and Challenge, first edition, 2010. doi:10.1002/9780470665251.ch17.

[16] M. Sheikh Shanawaz, N. Horta, A. G. Ravelo-García, F. Morgado-Dias, Analog active filter design using a multi objective genetic algorithm, AEU-International Journal of Electronics and Communications (2018).

[17] R. G. Mishra, R. Mishra, P. Kuchhal, N. P. Kumari, Optimization and analysis of high gain wideband microstrip patch antenna using genetic algorithm, International Journal of Engineering \& Technology 7 (1.5) (2018) 176-179.

[18] X. Qing, C. K. Goh, Z. N. Chen, Impedance characterization of RFID tag antennas and application in tag co-design, IEEE Transactions on Microwave Theory and Techniques 57 (5) (2009) 1268-1274. doi:10.1109/TMTT.2009.2017288.

[19] M. A. Ziai, J. C. Batchelor, Curved RFID tags for metallic gas cylinders, Electronics letters 45 (17) (2009) 867-869.

[20] G. Wu, J. Ouyang, B. Chen, L. Jin, R. Long, A small flexible antimetal RFID tag antenna, in: 2016 IEEE 5th Asia-Pacific Conference on Antennas and Propagation (APCAP), IEEE, 2016, pp. 289-290. 